\title{
Long non-coding RNASNHG17 promotes the progression of breast cancer by sponging miR-124-3p
}

Ye Du', Na Wei ${ }^{2}$, Jinghui Hong ${ }^{1}$ and Weiyun Pan $^{3^{*}}$ (D)

\begin{abstract}
Background: Small nucleolar RNA host gene 17 (SNHG17), a novel cancer-related long noncoding RNA (IncRNA), was reported to be responsible for processing and developing in several cancers. Nonetheless, the clinical significance and biological function of SNHG17 in human breast cancer (BC) remain rarely known.

Materials and methods: 58 pairs of $B C$ tissues and adjacent non-cancerous tissues were harvested to measure SNHG17 expression levels. SNHG17 was knockdown to study its biological behavior in BC cells. The microRNAs (miRNAs) that can bind to SNHG17 were predicated using Starbase2.0 and were tested using luciferase reporter activity and RIP assays. A xenograft model was established to investigate the impact of SNHG17 in tumor growth in vivo.
\end{abstract}

Results: An increased SNHG17 was observed in BC samples and cell lines compared with corresponding control. Increased SNHG17 was closely associated with poor prognosis.SNHG17 depletion suppressed cell proliferation, migration and invasion in vitro, as well as inhibited tumor growth in xenograft tumor models. Mechanistically, SNHG17 could function as an endogenous sponge of miR-124-3p in BC cells. Moreover, the repression of cell proliferation, migration and invasion induced by SNHG17 knockdown would reversed by miR-124-3p inhibitor.

Conclusion: The present study demonstrated that the IncRNASNHG17 could regulate the progression of BC by sponging miR-124-3p.

Keywords: Breast cancer, SNHG17, miR-124-3p

\section{Background}

Breast cancer $(\mathrm{BC})$ is one of the most prevalent types of malignancy and a leading cause of cancer-related death among women worldwide [1]. Despite recent advances in surgery, chemotherapy and radiotherapy, the prognosis of $\mathrm{BC}$ remains poor because of its heterogeneous nature and metastasis to other organs $[2,3]$. Therefore, there is an urgent need to explore molecule mechanism involved in $\mathrm{BC}$ progression to develop new therapeutic targets for this cancer.

*Correspondence: panweiyuan108@126.com

${ }^{3}$ Department of ICU, The First Hospital of Jilin University,

Changchun 130021, Jilin, People's Republic of China

Full list of author information is available at the end of the article
In recent years, non-coding RNAs (ncRNAs) have gained widely attention as studies have shown that they are involved in various critical pathophysiological processes, including cell proliferation, metastasis and invasion, and so on $[4,5]$. The ncRNAs includes long noncoding RNAs (lncRNAs, a type of ncRNAs over 200 nucleotides in length and with limited protein-coding ability) [6], and microRNAs that inhibit target gene expression by binding to the $3^{\prime}$ UTR ( $3^{\prime}$ untranslated region) of a target gene [7]. Both lncRNAs and miRNAs were shown to be involved in the regulation of the initiation and development of various cancers including $\mathrm{BC}$ $[8,9]$. A body of evidence demonstrated that lncRNAs could serve as competing endogenous RNAs (ceRNAs)

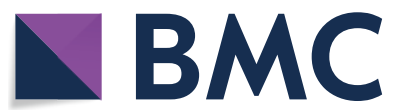

(c) The Author(s) 2020. This article is licensed under a Creative Commons Attribution 4.0 International License, which permits use, sharing, adaptation, distribution and reproduction in any medium or format, as long as you give appropriate credit to the original author(s) and the source, provide a link to the Creative Commons licence, and indicate if changes were made. The images or other third party material in this article are included in the article's Creative Commons licence, unless indicated otherwise in a credit line to the material. If material is not included in the article's Creative Commons licence and your intended use is not permitted by statutory regulation or exceeds the permitted use, you will need to obtain permission directly from the copyright holder. To view a copy of this licence, visit http://creativeco mmons.org/licenses/by/4.0/. The Creative Commons Public Domain Dedication waiver (http://creativecommons.org/publicdomain/ zero/1.0/) applies to the data made available in this article, unless otherwise stated in a credit line to the data. 
for miRNAs to regulate miRNAs and miRNA-targeted mRNAs [10].

Small nucleolar RNA Host Gene 17 (SNHG17), an important lncRNA, was proven to play oncogenic role in non-small cell lung cancer [11], gastric cancer [12], colorectal cancer [13] and melanoma [14]. However, the biological function of SNHG17 in the initiation and development of $\mathrm{BC}$ remains poorly understood. Therefore, we strive to elucidate the functional roles of SNHG17 in BC. We found that knockdown of SNHG17 inhibited the growth of $\mathrm{BC}$ in vivo and in vitro. We also selected miRNAs that bind to SNHG17 using starbase2.0, and identified that miR-124-3p, a known tumor-suppressive miRNA in $B C$ [15-18], was a direct target of miR124-3p. We investigate whether the SHNG17 regulate the progression of $\mathrm{BC}$ by sponging miR-124-3p.

\section{Materials and methods}

\section{Clinical tissue specimens}

A total of 58 pairs of BC tissues and paired non-tumor tissues were collected from the First Hospital of Jilin University from Jan 2013 to Jan 2014. All the experiments concerning the use of patient samples in this stud were conducted in accordance with the Declaration of Helsinki and were approved by the Medical Ethics Committee of Jilin University. Written informed consents were acquired from all patients. All tissues were stored at in liquid nitrogen. The clinical features of the patients with $\mathrm{BC}$ were listed in Table 1.

\section{Cell lines and culture}

Human normal human breast epithelial cell (MCF-10A) and four breast cancer cell lines (MCF-7, MDA-MB-231, MDA-MB-468 and BT-474) were purchased from the ATCC (Manassas, VA, USA). L-15 medium (Gibco, CA, USA) supplemented with $10 \%$ FBS (fetal bovine serum, Gibco) was used to culture the cells in a high humidity incubator $\left(37^{\circ} \mathrm{C} ; 5 \% \mathrm{CO}_{2}\right)$.

\section{Virus infection and cell transfection}

Recombinant lentiviruses expressing the sh-SNHG17 (shRNA directly targeting SNHG17; 5'-GAUUGUCAG CUGACCUCUGUCCUGU-3') and sh-NC (negative control shRNA; 5'-UUCUCCGUUCGUGUCACGUUU$3^{\prime}$ ) were bought from Sangon Biotech Company (Shanghai, China). MCF-7 and MDA-MB-231 were transfected with sh-NC or sh-SNHG17 lentiviral transduction particles $(\mathrm{MOI}=20)$ using $5 \mu \mathrm{g} / \mathrm{mL}$ polybrene (Genechem). Stably transfected cells was chosen with $1 \mu \mathrm{g} / \mathrm{ml}$ puromycin (Calbiochem, USA) for 2 weeks. The mimics and inhibitor of miR-124-3p and their controls (miR-NC) were bought by GenePharma (Shanghai, China), and were transfected with MCF-7 and MDA-MB-231 cells

\begin{tabular}{|c|c|c|c|c|}
\hline \multirow[t]{2}{*}{ Variables } & \multirow[t]{2}{*}{ No. of cases } & \multicolumn{2}{|c|}{$\begin{array}{l}\text { SNHG17 } \\
\text { expression }\end{array}$} & \multirow[t]{2}{*}{$P$ value } \\
\hline & & High & Low & \\
\hline Age (years) & & & & $P=0.5936$ \\
\hline$<50$ & 22 & 11 & 11 & \\
\hline$\geq 50$ & 36 & 21 & 15 & \\
\hline ER & & & & $P=0.1103$ \\
\hline Positive & 34 & 22 & 12 & \\
\hline Negative & 24 & 10 & 14 & \\
\hline TNM stage & & & & $P=0.0014$ \\
\hline$|-| \mid$ & 44 & 19 & 25 & \\
\hline III-IV & 14 & 13 & 1 & \\
\hline Tumor size & & & & $P=0.2685$ \\
\hline$\leq 2 \mathrm{~cm}$ & 40 & 20 & 20 & \\
\hline$>2 \mathrm{~cm}$ & 18 & 12 & 6 & \\
\hline Lymph node metastasis & & & & $P=0.0249$ \\
\hline No & 45 & 21 & 24 & \\
\hline Yes & 13 & 11 & 2 & \\
\hline
\end{tabular}

using Lipofectamine 2000 (Invitrogen) in 6-well plates when cells grown to $70 \%$ confluent.

\section{Quantitative real-time polymerase chain reaction (qR-TPCR)}

Trizol reagent kit (Invitrogen) was applied to isolate total RNA from cultured cells or tissues. The concentration and purify of RNA was determined using Nano-Drop 2000 spectrophotometer (Termo Scientific, USA). The PrimeScript $^{\mathrm{TM}}$ RT reagent Kit (Takara, Japan) was used to synthesis cDNA. The expression of miR-124-3p was determined using miRNA qRT-PCR Starter kit (Riobobio, USA) using primers of U6 and miR-124-3p (Riobobio). SYBR ${ }^{\circledR}$ Green Real time PCR Msater Mix (Toyobo, Japan) was used to detect SNHG17 expression. Expression levels of miR-124-3p and SNHG17 were normalized by U6 and GADPH, respectively. The relative expression of miR-124-3p and SNHG17 was calculated by using $2^{-\Delta \Delta \mathrm{Ct}}$ method.

\section{RNA isolation of nuclear and cytoplasmic fractions}

The NE-PER ${ }^{\mathrm{TM}}$ Nuclear and Cytoplasmic Extraction Reagents Kit (Thermo Scientific, USA) was used to isolate and collect cytosolic and nuclear fractions according to manufacturer's protocol. The expression levels of SNHG17, U6 (nuclear control transcript) and GAPDH (cytoplasmic control transcript) were examined in cytoplasmic and nuclear fractions using qRT-PCR as described above-mentioned. 


\section{Cell proliferation assay}

Cell Counting Kit-8 (CCK-8; Dojindo, Japan) and colony formation assays were used to determine cell proliferation. In CCK-8 assay, stable transfected cells were collected and seeded into 96-well plates at a den-sity of $1 \times 10^{4}$ cells per well. After 24,48 or $72 \mathrm{~h}$ (hs), cells was treated using $10 \mu \mathrm{l} \mathrm{CCK-8}$ assay for $2 \mathrm{~h}$. The proliferation ability was determined by measuring absorbance at $450 \mathrm{~nm}$ using an enzyme immunoassay analyzer (Biorad, Hercules, CA, USA).

For colony formation assay, 1000 stable transfected cells were added into six-well plates and cultured for 10 days. After washed with PBS, colonies were fixed with methanol and stained with $0.1 \%$ crystal violet (SigmaAldrich, StLouis, MO, USA) for $15 \mathrm{~min}$. The visible colonies were counted.

\section{Cell migration and invasion assays}

Wound healing assay used to assess cell migration ability as described previously [19]. Stable transfected cells were seeded in six-well plates until full confluence. The monolayer cells were manually scraped to create wound area using a $100 \mu \mathrm{l}$ sterile pipette tip. The wounding area at $0 \mathrm{~h}$ and $24 \mathrm{~h}$ was measured and imaged a light micro-scope (Olympus, Tokyo, Japan).

Transwell invasion assay was performed to assess cell invasion. Briefly, stably transfected cells were suspended in serum-free medium and added to upper transwell chambers (BD Biosciences, Sparks, MD, USA) pre-coated with BD BioCoatMatrigel. After incubation $24 \mathrm{~h}$, invasive cells were fixed with methanol, stained with $0.1 \%$ crystal violet, and counted at five randomly selected fields in each well using a light micro-scope (Olympus).

\section{Luciferase reporter assay}

Oligonucleotides that encode SNHG17 cDNA encompassing miR-124-3p-binding sites were synthesized and inserted into pGL3 vector (WT-SNHG17).We also constructed a pGL3-Report plasmid that carried the mutant SNHG17 binding region (MUT-SNHG17). For the luciferase reporter assay, MCF-7 and MDA-MB-231cells were co-transfected with luciferase reporter plasmids and negative control (miR-NC) or miR-124-3p mimics using Lipofectamine 2000. A double-luciferase assay system (Beyotime, Wuhan, China) was used to determine the luciferase activity at $24 \mathrm{~h}$ post-transfection.

\section{RNA-binding protein immunoprecipitation (RIP) assay}

MCF-7 and MDA-MB-231 cells transfected with miR124-3p mimics or miR-NC were harvested and subjected to RIP assay using an anti-Ago2 antibody and a RIP kit (Millipore Inc., Bedford, MA, USA) according to the manufacturer's instructions. Mouse anti-human immunoglobulinG ( $\mathrm{IgG}$ ) antibody was used as control. Finally, RNA was extracted and quantified by qRT-PCR to examine the expression of SNHG17 as described above-mentioned.

\section{Animal experiment}

Female athymic BALB/c nude mice (5 weeks old, 18-20 g) were bought from Laboratory Animal center of Jilin University. All of the animal experiments were performed based on experimental protocols and approved by the Institutional Animal Care and Use Committee of the Jilin University.

To initiate tumor xenografts, $2 \times 10^{6}$ MCF- 7 cells that were stably transfected with sh-NC or sh-SNHG17 were subcutaneously injected into the flank of mice. Tumor growth was measured by caliper measurements every week and was calculated based on following formula: volume $=0.5 \times$ length $\times$ width $\times$ width. After 5 weeks, all mice were euthanized by intraperitoneal injection of $200 \mathrm{mg} / \mathrm{kg}$ pentobarbital. Tumor tissues were removed and weighed. A part of tumor was used for immunohistochemical staining to detect the expression of Ki-67 (a proliferation marker) as described previously [20].

\section{Statistical analysis}

All results are presented as the mean $\pm \mathrm{SD}$ of at least three independent experiments, are analyzed using the SPSS20.0 statistical software package. Student's t-tests and one-way ANOVA were used to determine statistically significant differences between groups. The Chisquare test was used to analyze the correlation between the expression of SNHG17 and clinical parameters of patients with BC. Kaplan-Meier method and a log-rank test were used to assess overall survival rate. Spearman's correlation coefficient was used to assess correlation between two groups. A $P$ value $<0.05$ was considered statistically significant.

\section{Results \\ Upregulation of SNHG17 is associated with poor prognosis of $B C$ patients}

To evaluate the expression pattern of SNHG17 in BC, we first examined the expression of SNHG17 in the BC tissues and adjacent normal tissues. As shown in Fig. 1a, the expression of SNHG17 was upregulated in BC tissues compared with non-tumor breast tissues. To assess the association with clinical characteristic of $\mathrm{BC}$ patients and SNHG17 expression, we divided the patients to highexpression group $(\mathrm{n}=32)$ and low-expression group $(n=26)$ based on the median level of SNHG17 expression. Table 1 displayed that increasedSNHG17 expression was positively associated with advanced TNM stages 

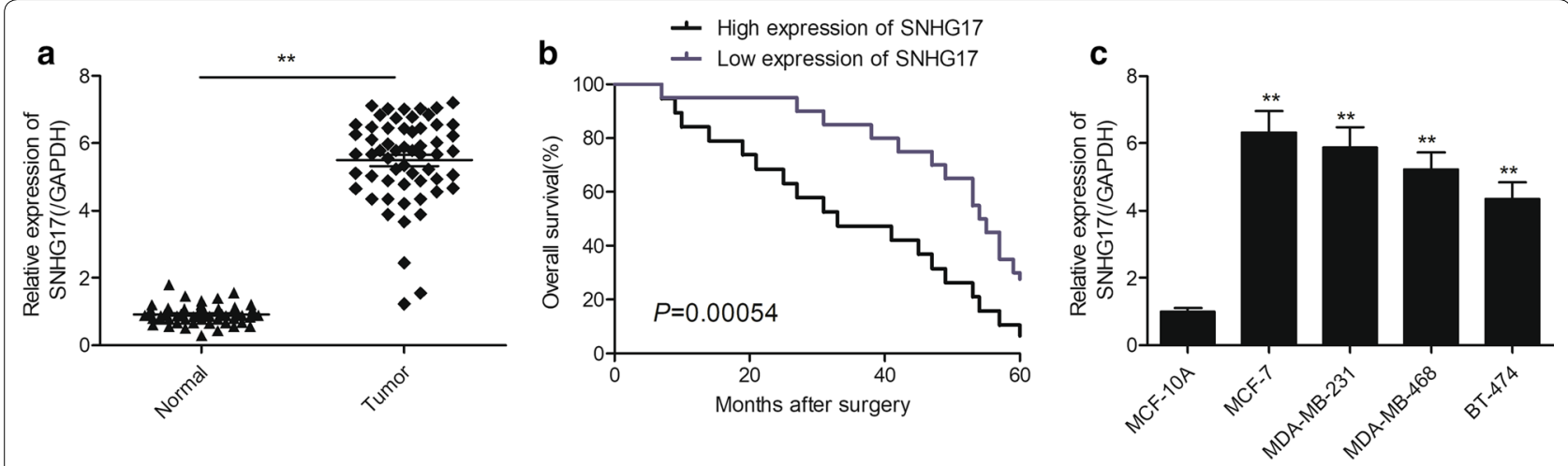

Fig. 1 SNHG17 is upregualted in BC tissues and correlated with poor outcomes in patients with BC. a Relative expression of SNHG17 in 58 BC tissues and corresponding adjacent normal breast tissues. b Kaplan-Meier overall survival curves based on SNHG17 expression levels. c SNHG17 expression in human normal human breast epithelial cell (MCF-10A) and four breast cancer cell lines. ${ }^{*} P<0.05$, ${ }^{* *} P<0.01$

(III-IV stages) and lymph node metastasis. KaplanMeier analyses revealed that high SNHG17 expression group has poorer survival than in low SNHG17 expression group (Fig. 1b). In addition, we found that SNHG17 expression was increased in four $\mathrm{BC}$ cell lines compared to MCF-10A cells (Fig. 1c).

\section{SNHG17 knockdown inhibits tumor growth of $B C$ in vitro and in vivo}

To investigate the role of SNHG17 in BC cells, loss-of function experiments were performed in MCF-7 and MDA-MB-231 cells by transfection with shRNA against SNHG17 plasmid (sh-SNHG17). We found that transfection of sh-SNHG17 was able to decrease SNHG17 expression in MCF-7 and MDA-MB-231 cells (Fig. 2a). CCK8 assay revealed that knockdown of SNHG17 could result in a significant decrease of cell proliferation in MCF-7 and MDA-MB-231 cells (Fig. 2b). Consistent with this result, silence of SNHG17 inhibited colony formation of MCF-7 and MDA-MB-231 cells (Fig. 2c).

To investigate whether SNHG17 depletion affect the tumor growth in vivo, the xenograft tumor were established. We found that xenografts grew more slowly in SNHG17 depletion group than in sh-NC group (Fig. 2d). Moreover, the weight and size of tumors was decreased in the SNHG17 depletion group compared with those in the sh-NC group (Fig. 2e). More importantly, the Ki-67 expression was reduced in SNHG17 depletion group compared with sh-NC group (Fig. 2f). These results suggested that SNHG17 depletion inhibited BC growth in vitro and in vivo.

\section{SNHG17 knockdown inhibits migration and invasion of BC cells}

Next, we checked the effects of SNHG17 knockdown on cell invasion and migration using the transwell assay and wound healing assay, respectively. The migration and migration capabilities were significantly decreased in MCF-7 and MDA-MB-231 cells transfection with shSNHG17 compared with cells transfected with sh-NC (Fig. 3a, b).

\section{miR-124-3p is a target of SNHG17 in BC cells}

To elucidate whether SNHG17 functioned as a ceRNA in regulating $\mathrm{BC}$ progression, Starbse2.0 software (http:// starbase.sysu.edu.cn/starbase2/index.php) was used to predict potential target miRNAs of SNHG17. Among the candidates, miR-124-3p, a tumor suppressor miRNA that is frequently observed to be downregulated in $\mathrm{BC}$ [15-18], was selected and further study. As illustrated in Fig. 4a, there is a predicted targeting site of miR-124-3p on SNHG17. To test this predication, luciferase activity was performed. Our result demonstrated that miR124-3p mimics could inhibit the luciferase activity in WT-SNHG17 group, with no effect in MT-SNHG17 group in MCF-7 and MDA-MB-231 cells (Fig. 4b). Moreover,we ascertain the subcellular localization of SNHG17 in MCF-7 and MDA-MB-231 cells. As illustrated in Fig. 4c, SNHG17 was mainly expressed in the cytoplasm of MCF-7 and MDA-MB-231 cells.RIP assays further demonstrated that miR-124-3p and SNHG17 were all enriched in Ago2-immunoprecipitation (Ago2IP) relative to the control in MCF-7 and MDA-MB-231 cells (Fig. 4d). In addition, we found that knockdown of SNHG17 significantly increased miR-124-3p expression in MCF-7 and MDA-MB-231 cells (Fig. 4e), while overexpression of miR-124-3p led to a significant decrease in both MCF-7 and MDA-MB-231 cells (Fig. 4f). We also found that SNHG17 levels were negatively correlated with miR-124-3p levels in BC tissues (Fig. 4g). These results suggested that miR-124-3p bound to the transcript position of SNGH17. 


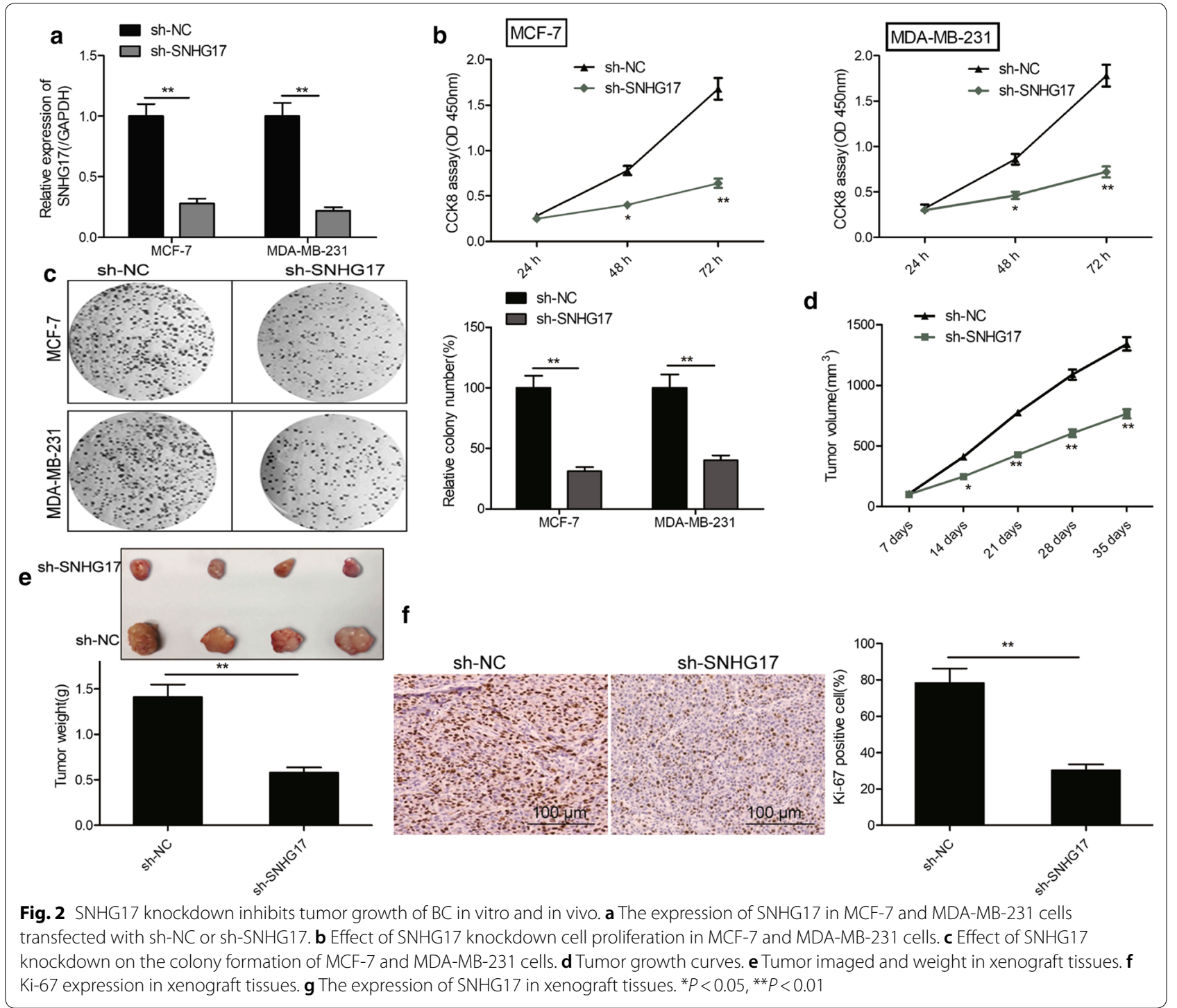

\section{SNHG17 knockdown inhibits the progression of BC cells} by regulating miR-124-3p

Considering the close correlation between miR-124-3p and SNHG17, we next evaluated whether the miR124-3p expression implicates in biological effects by SNHG17 in BC cells. To this end, MCF-7 and MDAMB-231 were transfected with sh-NC, sh-SNHG17 and sh-SNHG1 + miR-124-3p inhibitor. We found that transfection with sh-SNHG17 obviously increased miR124-3p expression in MCF-7 and MDA-MB-231 cells, while transfection of miR-124-3p inhibitor partially reversed this trend (Fig. 5a). In addition,miR-124-3p inhibitor partially reversed the inhibitory effect on cell proliferation, colony formation, invasion and migration caused by SNHG17 knockdown in BC cells (Fig. 5b-e).
In summary, these findings suggested that SNHG17 promoted $\mathrm{BC}$ growth and metastasis via modulation of miR-124-3p (Fig. 5f).

\section{Discussion}

Multiple lncRNAs have been identified to serve as crucial modulators in modulating the progression of various cancers including $\mathrm{BC}[21,22]$. Zhu et al. revealed that lncRNA linc00460 drove $\mathrm{BC}$ progression via regulating the miR-489-5p/FGF7/AKT axis [23]. Yang et al. reported that IncRNAADPGK-AS1 promotes BC cell proliferation, migration, and epithelial-mesenchymal transition (EMT) process through regulating miR-3196/OTX1 axis [24]. Sun et al. demonstrated that SNHG7 promoted proliferation, invasion and EMT initiation of $\mathrm{BC}$ by sponging 

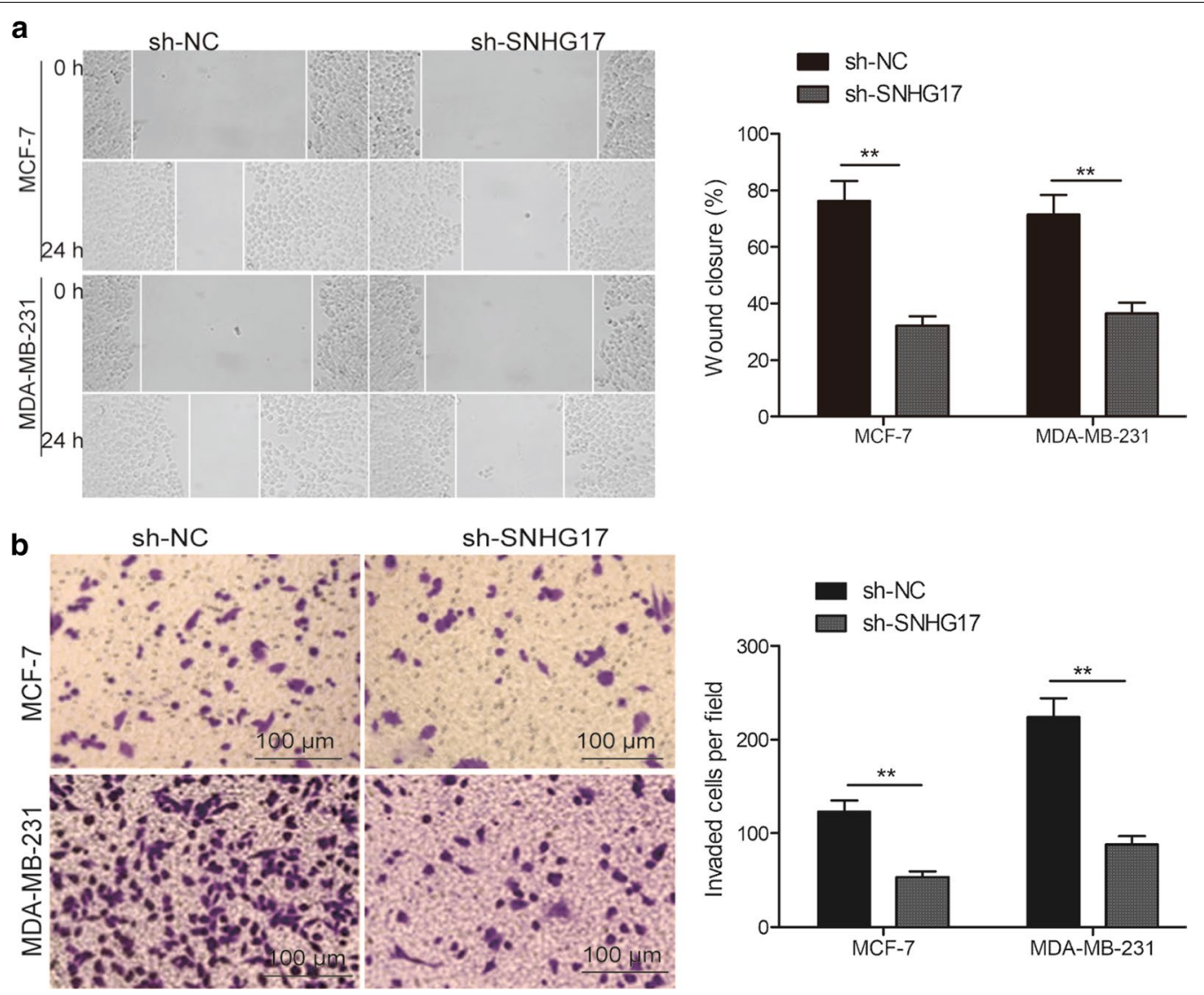

Fig. 3 SNHG17 knockdown inhibits migration and invasion of BC cells. a The migration in MCF-7 and MDA-MB-231 cells transfected with sh-NC or sh-SNHG 17. b The invasion in MCF-7 and MDA-MB-231 cells transfected with sh-NC or sh-SNHG17. ${ }^{*} P<0.05,{ }^{*} P<0.01$

miR-34a and regulating Notch-1 pathway [25]. Here, we indicated lncRNA SNHG17 was increased expression in $\mathrm{BC}$ cell lines and tissues, and was closely associated with lymph node metastasis, advanced TNM stage, and poor overall survival ratio. Additionally, knockdown of SNHG17 inhibited BC cell proliferation, invasion and migration by regulating miR-124-3p.

Aberrant expression of SNHG17 has been reported to play oncogenic role in several cancers by regulating cancer cell proliferation, migration and metastasis $[11-13,26]$. However, the role of SNHG17 in BC is not well known. Here, for the first time, we investigate the expression of SNHG17 expression in BC tissues and cell lines, and found that SNHG17 expression was upregualted and was associated with poor prognosis. Functional assay showed that downregulation of SNHG17 significantly inhibited cell proliferation and migration in vitro, and suppressed tumor growth in vivo. These results implied that SNHG17 plays an oncogenic role in $\mathrm{BC}$ progression.

Accumulating evidence illuminated that IncRNAs exerted biological various roles in various cancer by functioning as ceRNAs for sponging miRNA to regulating miRNAs expression [27]. Here, we found that SNHG17 expression was mainly enriched in the cytoplasm fraction of BC cells, indicating SNHG17 could interact with miRNAs. Through bioinformatics analysis, luciferase activity and RIP assays, we determined that miR-124-3p is a sponge of SNHG17 in BC cells.miR-124-3p has been to exhibit a low expression level and play a tumor suppressive role in $B C$ by regulating multiple genes [15-18].Here, our result showed that miR-124-3p expression was negative correlated with SNHG17 expression in BC tissues. Based on rescue assays, we confirmed that miR-124-3p involved in SNHG17-mediated BC progression. These results implied that SNHG17 exerted an oncogenic role 

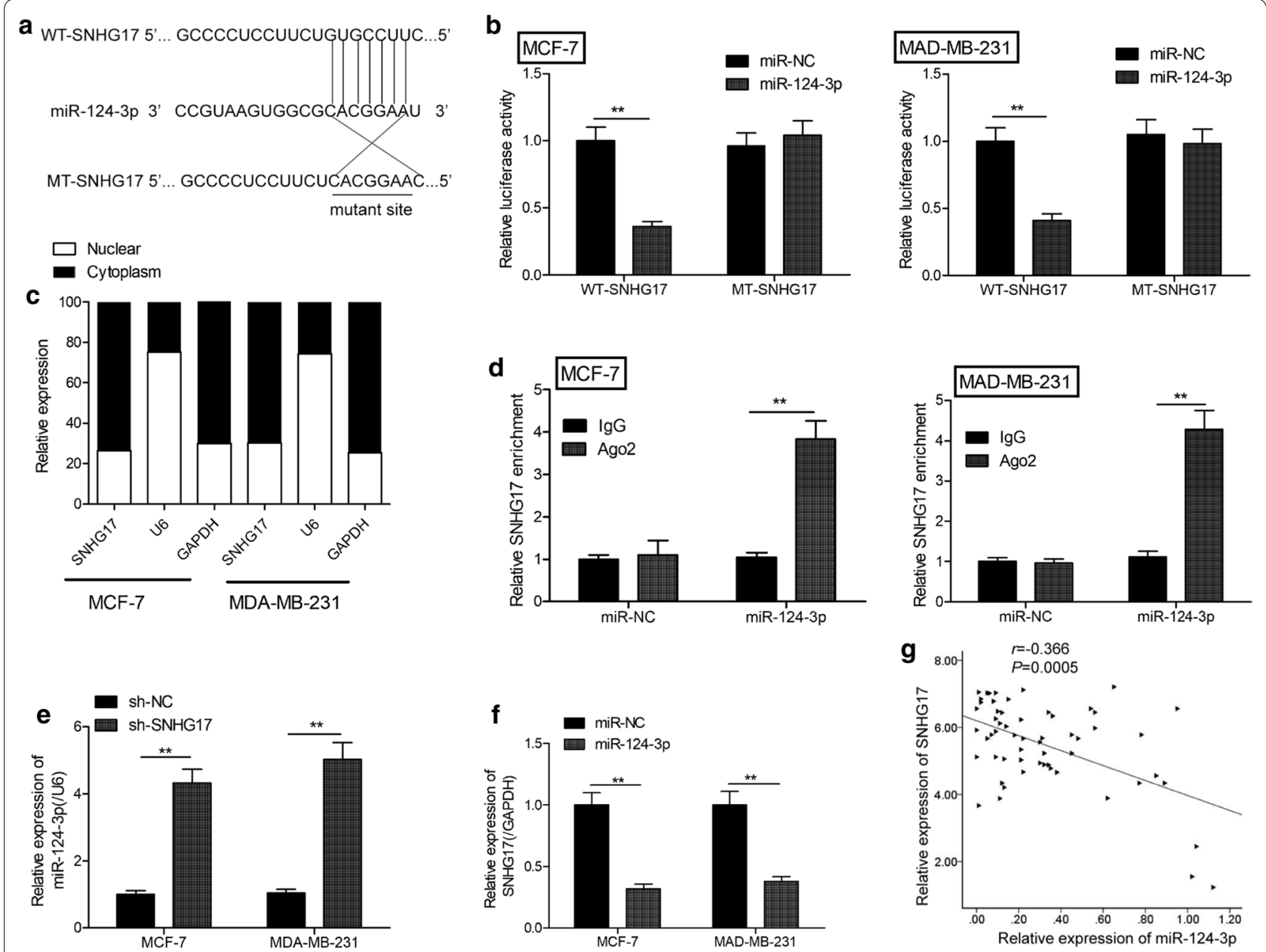

Fig. 4 miR-124-3p is a target of SNHG17 in BC cells. a The predicted binding sites of miR-124 on the sequence of SNHG17 (WT-SNHG17). The target sequences of the SNHG17 were mutated (MT-SNHG17). b Luciferase activity in MCF-7 and MDA-MB-231 cells co-transfected with miR-124-3p mimics or miR-NC, and luciferase reporter vector containing WT-SNHG17 or MT-SNHG17. WT wild-type, MT mutant-type. c The expression of SNHG17 in nuclear and cytoplasmic of MCF-7 and MDA-MB-231 cells by qRT-PCR. $\mathbf{d}$ The interaction between miR-124-3p and SNHG17 in MCF-7 and MDA-MB-231 cells were tested by RIP experiment. e The expression of miR-124-3p in MCF-7 and MDA-MB-231 cells transfected with sh-NC or sh-SNHG17. f The expression of SNHG17 in MCF-7 and MDA-MB-231 cells transfected with miR-NC or miR-124-3p mimics. $\mathbf{g}$ Spearman's correlation coefficient analysis between miR-124-3p expression and SNHG17 expression in 58 patients with $B C$. ${ }^{*} P<0.05,{ }^{*} P<0.01$

in $\mathrm{BC}$ partially by regulating miR-124-3p. However, miR124-3p could regulate multiple targets genes, we would investigate whether SNHG17 could modulate these target genes in $\mathrm{BC}$ to further explore molecular mechanism of SNHG17 in this cancer.

\section{Conclusion}

In conclusion, through integrating clinical data, we for the first time reveal that the SNHG17 is upregulated in $\mathrm{BC}$ tissues and is associated with poor prognosis. Through functional experiments, we showed that SNHG17 serve as an oncogene in BC facilitating cell 


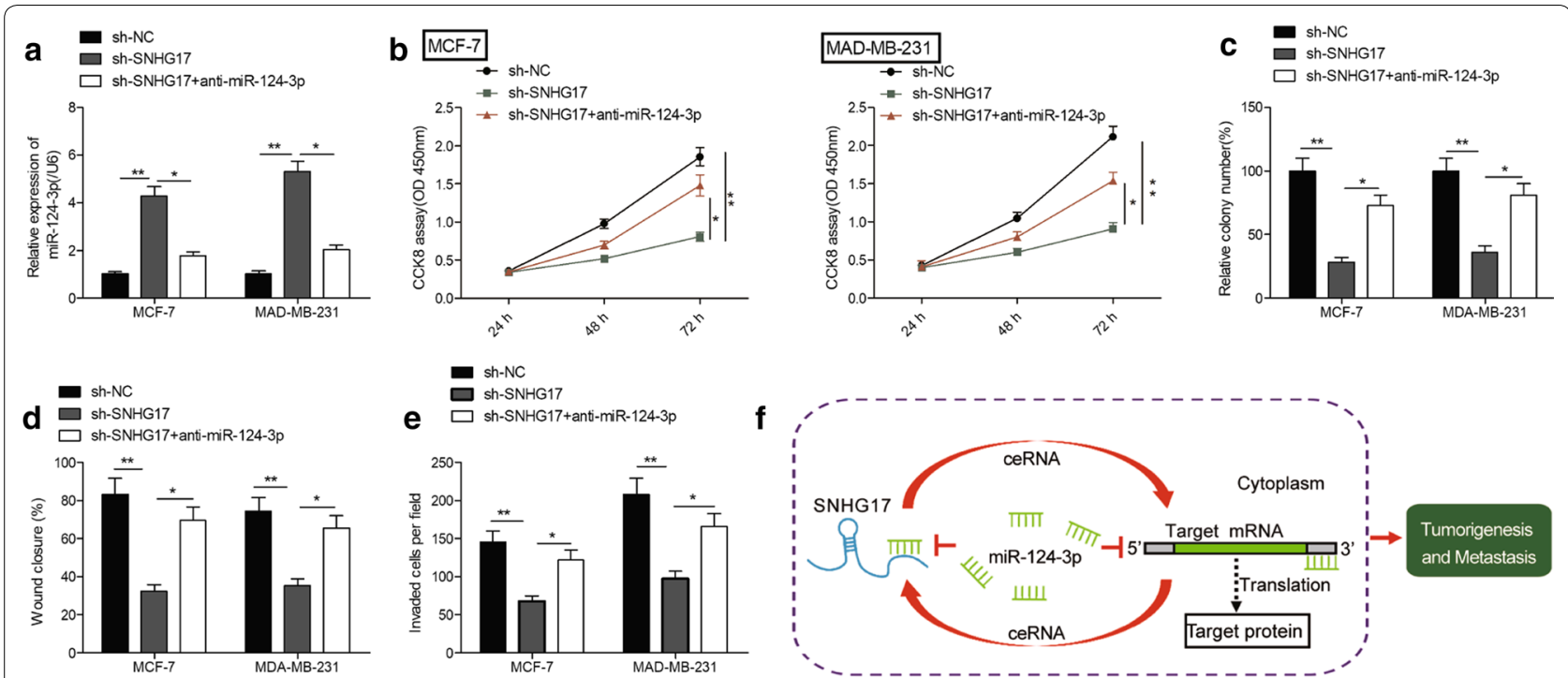

Fig. 5 SNHG17 knockdown inhibits the progression of BC cells by regulating miR-124-3p. a The expression of miR-124-3p in MCF-7 and MDA-MB-231 cells transfected with sh-NC, sh-SNHG17 and sh-SNHG17 + miR-124-3p inhibitor(anti-miR-124-3p). b-e Cell proliferation, colony formation, migration and invasion in MCF-7 and MDA-MB-231 cells transfected with sh-NC, sh-SNHG17 and sh-SNHG17+anti-miR-124-3p. f The schematic diagram of the mechanism of SNHG17/miR-124-3p axis in breast cancer. ${ }^{*} P<0.05,{ }^{* *} P<0.01$

proliferation, invasion and migration in vitro, as well as affecting tumor growth in vivo by sponging miR124-3p, highlighting a clinical potential of SNHG17 as a novel biomarker and potential therapeutic target for $\mathrm{BC}$.

\section{Abbreviations}

BC: Breast cancer; SNHG17: Small nucleolar RNA host gene 17; cDNA: Complementary DNA; CCK-8: Cell counting kit-8; IHC: Immunohistochemistry; RIP: RNA-binding protein immunoprecipitation (RIP); WT: Wile type; MT: Mutant type.

\section{Acknowledgements}

Not applicable.

\section{Authors' contributions}

The concept of the paper was devised and the first draft was written by WP. The experiment was done by $\mathrm{YD}, \mathrm{NW}$ and $\mathrm{JH}$ analyzed the data. All authors read and approved the final manuscript.

\section{Funding}

This study was supported by Jilin Province Department of Science and Technology (No. 20180520055JH)

\section{Availability of data and materials}

The data sets used and/or analyzed during the current study are available from the corresponding author upon reasonable request.

\section{Ethics approval and consent to participate}

This research was approved by the independent ethics committee of Jilin University, Changchun, P.R. China, and all patients provided written informed consent, in accordance with the Declaration of Helsinki.

\section{Consent for publication}

Not applicable.

\section{Competing interests}

The authors declare that they have no competing interests.

\section{Author details}

${ }^{1}$ Department of Breast Surgery, The First Hospital of Jilin University, Changchun 130021, Jilin, People's Republic of China. ${ }^{2}$ Department of First Operating Room, The First Hospital of Jilin University, Changchun 130021, Jilin, People's Republic of China. ${ }^{3}$ Department of ICU, The First Hospital of Jilin University, Changchun 130021, Jilin, People's Republic of China.

Received: 19 November 2019 Accepted: 29 January 2020

Published online: 05 February 2020

\section{References}

1. Bray F, Ferlay J, Soerjomataram I, Siegel RL, Torre LA, Jemal A. Global cancer statistics 2018: GLOBOCAN estimates of incidence and mortality worldwide for 36 cancers in 185 countries. CA Cancer J Clin. 2018;68(6):394-424.

2. Ganz PA, Goodwin PJ. Breast cancer survivorship: where are we today? Adv Exp Med Biol. 2015;862:1-8.

3. Nagini S. Breast cancer: current molecular therapeutic targets and new players. Anticancer Agents Med Chem. 2017;17(2):152-63.

4. Zhang P, Wu W, Chen Q, Chen M. Non-Coding RNAs and their Integrated Networks. J Integr Bioinform. 2019. https://doi.org/10.1515/jib-2019-0027.

5. Wang WT, Han C, Sun YM, Chen TQ, Chen YQ. Noncoding RNAs in cancer therapy resistance and targeted drug development. J Hematol Oncol. 2019;12(1):55.

6. Huarte M. The emerging role of IncRNAs in cancer. Nat Med. 2015;21(11):1253-61.

7. Bartel DP. MicroRNAs: genomics, biogenesis, mechanism, and function. Cell. 2004;116(2):281-97.

8. Tomar D, Yadav AS, Kumar D, Bhadauriya G, Kundu GC. Non-coding RNAs as potential therapeutic targets in breast cancer. Biochim Biophys Acta Gene Regul Mech. 2019. https://doi.org/10.1016/j.bbagr m.2019.04.005. 
9. $\quad$ Tang S, Fan W, Xie J, Deng Q, Wang P, Wang J, Xu P, Zhang Z, Li Y, Yu M. The roles of ncRNAs in the diagnosis, prognosis and clinicopathological features of breast cancer: a systematic review and meta-analysis. Oncotarget. 2017;8(46):81215-25

10. Tay Y, Rinn J, Pandolfi PP. The multilayered complexity of ceRNA crosstalk and competition. Nature. 2014;505(7483):344-52.

11. Xu T, Yan S, Jiang L, Yu S, Lei T, Yang D, Lu B, Wei C, Zhang E, Wang Z. Gene Amplification-driven long noncoding RNA SNHG17 regulates cell proliferation and migration in human non-small-cell lung cancer. Mol Ther Nucleic Acids. 2019;17:405-13.

12. Zhang G, Xu Y, Wang S, Gong Z, Zou C, Zhang H, Ma G, Zhang W, Jiang P. LnCRNA SNHG17 promotes gastric cancer progression by epigenetically silencing of p15 and p57. J Cell Physiol. 2019;234(4):5163-74.

13. Ma Z, Gu S, Song M, Yan C, Hui B, Ji H, Wang J, Zhang J, Wang K, Zhao Q. Long non-coding RNA SNHG17 is an unfavourable prognostic factor and promotes cell proliferation by epigenetically silencing P57 in colorectal cancer. Mol Bio Syst. 2017;13(11):2350-61.

14. Gao H, Liu R, Sun X. STAT3-induced upregulation of IncRNA SNHG17 predicts a poor prognosis of melanoma and promotes cell proliferation and metastasis through regulating PI3K-AKT pathway. Eur Rev Med Pharmacol Sci. 2019;23(18):8000-10.

15. Yan G, Li Y, Zhan L, Sun S, Yuan J, Wang T, Yin Y, Dai Z, Zhu Y, Jiang Z, et al. Decreased miR-124-3p promoted breast cancer proliferation and metastasis by targeting MGAT5. Am J Cancer Res. 2019;9(3):585-96.

16. Zhang L, Chen X, Liu B, Han J. MicroRNA-124-3p directly targets PDCD6 to inhibit metastasis in breast cancer. Oncol Lett. 2018;15(1):984-90.

17. Wang Y, Chen L, Wu Z, Wang M, Jin F, Wang N, Hu X, Liu Z, Zhang CY, Zen $K$, et al. miR-124-3p functions as a tumor suppressor in breast cancer by targeting CBL. BMC Cancer. 2016;16(1):826.

18. Zhang F, Wang B, Long H, Yu J, Li F, Hou H, Yang Q. Decreased miR-124-3p expression prompted breast cancer cell progression mainly by targeting Beclin-1. Clin Lab. 2016;62(6):1139-45.

19. Kanojia D, Garg M, Saini S, Agarwal S, Parashar D, Jagadish N, Seth A Bhatnagar A, Gupta A, Kumar R, et al. Sperm associated antigen 9 plays an important role in bladder transitional cell carcinoma. PLoS ONE. 2013;8(12):e81348.

20. Dong Y, Zhang D, Cai M, Luo Z, Zhu Y, Gong L, Lei Y, Tan X, Zhu Q, Han S. SPOP regulates the DNA damage response and lung adenocarcinoma cell response to radiation. Am J Cancer Res. 2019;9(7):1469-83.

21. Youness RA, Gad MZ. Long non-coding RNAs: functional regulatory players in breast cancer. Noncoding RNA Res. 2019;4(1):36-44.

22. Pecero ML, Salvador-Bofill J, Molina-Pinelo S. Long non-coding RNAs as monitoring tools and therapeutic targets in breast cancer. Cell Oncol. 2019;42(1):1-12.

23. Zhu Y, Yang L, Chong QY, Yan H, Zhang W, Qian W, Tan S, Wu Z, Lobie PE, Zhu T. Long noncoding RNA Linc00460 promotes breast cancer progression by regulating the miR-489-5p/FGF7/AKT axis. Cancer Manag Res. 2019;11:5983-6001

24. Yang J, Wu W, Wu M, Ding J. Long noncoding RNA ADPGK-AS1 promotes cell proliferation, migration, and EMT process through regulating miR-3196/OTX1 axis in breast cancer. In Vitro Cell Dev Biol Anim. 2019;55(7):522-32.

25. Sun X, Huang T, Liu Z, Sun M, Luo S. LncRNA SNHG7 contributes to tumorigenesis and progression in breast cancer by interacting with miR34a through EMT initiation and the Notch-1 pathway. Eur J Pharmacol. 2019;856:172407

26. Chen LL, He J, Qiu XT, Yu J, Wang ZM. The prognostic roles of long noncoding RNA SNHG17 in the patients with gastric cancer. Eur Rev Med Pharmacol Sci. 2019;23(3):1063-8.

27. Abdollahzadeh R, Daraei A, Mansoori Y, Sepahvand M, Amoli MM, Tavakkoly-Bazzaz J. Competing endogenous RNA (ceRNA) cross talk and language in ceRNA regulatory networks: a new look at hallmarks of breast cancer. J Cell Physiol. 2019;234(7):10080-100.

\section{Publisher's Note}

Springer Nature remains neutral with regard to jurisdictional claims in published maps and institutional affiliations.
Ready to submit your research? Choose BMC and benefit from:

- fast, convenient online submission

- thorough peer review by experienced researchers in your field

- rapid publication on acceptance

- support for research data, including large and complex data types

- gold Open Access which fosters wider collaboration and increased citations

- maximum visibility for your research: over 100M website views per year

At BMC, research is always in progress.

Learn more biomedcentral.com/submissions 